\title{
LINGUISTIQUE
}

\author{
BRUNON BULIKOWSKI
}

Université Adam Mickiewicz

\section{LES CRITĖRES DE CLASSEMENT DES CATÉGORIES GRAMMATICALES - UNE PERSPECTIVE DIACHRONIQUE}

\begin{abstract}
Bulikowski Brunon, Les critères de classement des catégories grammaticales - une perspective diachronique [The classification criteria of grammatical categories from a diachronic perspective], Studia Romanica Posnaniensia, Adam Mickiewicz University Press, Poznań, vol. XXVII: 2001, pp. 41-53. ISBN 83-232-1039-X, ISSN 0137-2475.

This paper deals with different ways of classifying grammatical categories, from the antiquity to the present. The author refers to the works of Aristotle as well as to ideas of American and European structural grammarians. A large part of this article is devoted to an explication of the medieval Modistes theory of speculative grammar. This paper is also concerned with the opposition between grammatical and logical criteria.
\end{abstract}

Les premières tentatives de typologie des catégories de la langue et de celles de la pensée remontent à l'Antiquité. Si nous nous penchons donc sur ce problème, nous ne pouvons pas oublier l'essai intitulé Catégories, qui ouvre le célèbre ouvrage d'Aristote, Organon.

Les opinions sur le caractère des catégories aristotéliciennes sont partagées. Par exemple, d'après J. Tricot, les dix catégories du philosophe grec ne sont nullement grammaticales, tandis que A. Rey souligne que la réflexion d'Aristote reste ambigué, ce qui "peut donner naissance à une réaction facile: celle qui consiste à affirmer qu'Aristote a »confondu« langue et pensée» (Rey, 1973: 22). Cependant, E. Benveniste, dans son article Catégories de pensée et catégories de langue, essaie de montrer que les distinctions faites par le philosophe grec dans l'Organon sont d'abord des catégories de langue, et qu'en fait, raisonnant d'une manière absolue, leur auteur retrouve simplement certaines des catégories fondamentales de la langue dans laquelle il pense (Benveniste, 1966: 66).

Citons d'abord une courte réflexion d'Aristote extraite du chapitre en question: «Chacune des expressions n'entrant pas dans une combinaison signifie: la substance; ou combien; ou quel; ou relativement à quoi; ou où; ou quand; ou être en posture; ou être en état; ou faire; ou subir. «Substance», par exemple, en général, «homme; cheval»; - «combien», par exemple «de deux coudées; de trois 
coudées» - «quel» par exemple «blanc; instruit»; - «relativement à quoi», par exemple «double; demi; plus grand»; - «où», par exemple «au Lycée; au marché»; «quand», par exemple «hier, l'an passé»; - «être en posture», par exemple «il est couché; il est assis»; - «être en état», par exemple «il est chaussé; il est armé»; - «faire», par exemple «il coupe; il brûle»; - «subir», par exemple «il est coupé; il est brûlé» (ibid.: 66)». Dans la traduction de l'Organon faite par Tricot, les dix catégories sont rendues par les termes: substance, quantité, qualité, relation, lieu, temps, position, possession, action, passion.

Les six premières catégories présentées ci-dessus se réfèrent toutes à des formes nominales. «Substance» est la catégorie donnant à la question «quoi?» la réponse: «homme» ou «cheval», donc des mots qui appartiennent à la classe linguistique des noms.

En ce qui concerne les expressions qui signifient «combien», elles ne visent pas que le nombre mais plus généralement tout ce qui est susceptible de mesure. La catégorie suivante englobe la «qualité» sans acception d'espèces: «homme ou animal (...) de telles expressions signifient plutôt une qualification, car le sujet n'est pas un comme dans le cas de la substance première; en réalité, homme est attribué à une multiplicité, et animal également. Cependant ce n'est pas d'une façon absolue que l'espèce et le genre signifient la qualité, comme le ferait, par exemple, le blanc (car le blanc ne signifie rien d'autre que la qualité), mais ils déterminent la qualité par rapport à la substance: ce qu'ils signifient, c'est une substance de telle qualité» (Catégories, chap.V). Selon Benveniste, à la catégorie de la qualité et à celle de la quantité citées ensemble répond non pas seulement la classe des adjectifs en général, mais spécialement deux types d'adjectifs que le grec associe étroitement.

Aristote se sert du terme «relatif» pour désigner «ces choses dont tout l'être consiste en ce qu'elles sont dites dépendre d'autres choses, ou se rapporter de quelque autre façon à autre chose: par exemple, le plus grand est ce dont tout l'être consiste à être dit d'une autre chose, car c'est de quelque chose qu'il est dit plus grand; et le double est ce dont tout l'être est d'être dit d'une autre chose, car c'est de quelque chose qu'il est dit le double» (ibid., chap.VII). La catégorie en question fournit donc non seulement un comparatif, mais de l'autre côté, elle sert à exprimer des concepts tels que celui de «double» ou de «demi» qui sont relatifs par définition. De plus, elle comprend ceux des adjectifs qualificatifs qui d'une façon se rapportent à une autre chose - «Ainsi, une montagne est dite grande par rapport à autre chose, car c'est par relation à une chose que la montagne est appelée grande» (ibid.).

$\mathrm{Vu}$ que les catégories du lieu et du temps, auxquelles correspondent respectivement les adverbes de lieu et de temps, ne nécessitent pas de commentaire, passons aux quatre dernières distinctions qui sont des catégories verbales. Celle de la position («être en posture») illustre la voix moyenne tandis que la catégorie de la possession («être en possession») traduit l'aspect perfectif. «Dans le système verbal du grec ancien, tel qu'il se maintient encore à l'époque classique, la véritable distinction est celle de l'actif et du moyen. Un penseur grec pouvait à bon droit 
poser dans l'absolu un prédicat qui s'énonçait par une classe spécifique de verbes, ceux qui ne sont que moyens (les media tantum), et qui indiquent entre autres la «posture», l'«attitude». Egalement irréductible à l'actif et au passif, le moyen dénotait une manière d'être aussi caractéristique que les deux autres» (Benveniste, 1966: 68). Les deux exemples grecs rendus en français par «il est couché» et «il est assis» sont des «spécimens de verbes moyens» (ibid.).

Pour mettre en évidence l'aspect perfectif des formes verbales qui représentent la catégorie de la possession (fr. «il est chaussé», «il est armé»), Benveniste propose l'interprétation suivante: «il a ses chaussures aux pieds», «il a ses armes sur lui». Le sens de cette catégorie rassemble à la fois le concept d' «avoir» et celui d'«être dans un état». Il faut ajouter ici que le parfait ne s'insère pas dans le système temporel du grec et reste à part, indiquant un mode de la temporalité ou une manière d'être du sujet (ibid.: 69).

Les deux dernières catégories, celles de l'action et de la passion englobent des expressions qui signifient respectivement «faire» et «subir», correspondant ainsi aux catégories de l'actif et du passif.

Benveniste termine son débat en démontrant, de manière définitive et irréfutable, que les catégories de la logique aristotélicienne ne sont, en réalité, que la transposition, en termes philosophiques, de catégories propres à la langue grecque. - «Pour autant que les catégories d'Aristote sont reconnues valables pour la pensée, elles se révèlent comme la transposition des catégories de langue. C'est ce qu'on peut dire qui délimite et organise ce qu'on peut penser. La langue fournit la configuration fondamentale des propriétés reconnues par l'esprit aux choses. Cette table des prédicats nous renseigne avant tout sur la structure des choses d'une langue particulière» (ibid.: 70).

Cet aspect de la théorie aristotélicienne a suscité des critiques de la part de linguistes contemporains. Relisons, à titre d'exemple, un court passage tiré de Langages de Leonard Bloomfield: «Les anciens Grecs n'étudiaient pas le langage mais le leur; ils considéraient comme admis que la structure de leur langage concrétisait les formes universelles de la pensée humaine ou, peut-être, l'ordre cosmique. En conséquence, ils faisaient des observations grammaticales, mais limitaient celles-ci à une seule langue et les énonçaient sous une forme philosophique. Ils aperçurent les parties du discours de leur langue, ses constructions syntaxiques, (...) et ses principales catégories inflexionnelles» (Bloomfield, 1970: 11).

Avant de clore la présentation des quelques aspects choisis de l'œuvre d'Aristote, considérons les remarques de ce philosophe sur ce qu'est le nom et le verbe. Les deux catégories sont examinées dans la Poétique et dans l'Herméneia. $\mathrm{Ce}$ qui distingue, selon Aristote, un nom d'un verbe, c'est le fait que le premier n'a pas d'idée de temps: «Le nom est un mot qui par convention signifie quelque chose sans spécifier de temps, et donc aucune partie séparée n'a de signification à elle (...) Le verbe est le mot qui, outre sa signification propre, embrasse l'idée de temps, et dont aucune partie isolée n'a de sens par elle-même (...)» (Herméneia, 1943: 149 et 
152). Tout verbe donc exprime à la fois: d'une part, une certaine idée particulière et, de l'autre, le temps dans lequel s'accomplit cette idée.

Aristote donne comme exemple de nom le mot ugíeia «santé» et comme exemple de verbe le mot ugiaínei «il a de la santé, il se porte bien». Il ajoute que le verbe indique toujours ce qui est dit sur autre chose: «[le verbe] est toujours le signe des choses attribuées à d'autres choses, par exemple: de choses dites d'un sujet ou qui sont dans un sujet» (ibid.). Il résulte de cette constatation que le verbe qui, au fond, est toujours le verbe substantif, est la copule du sujet et de l'attribut.

D'après Aristote, le verbe proprement dit est celui qui exprime le présent. La chose désignée «est» alors, tandis qu'ayec le passé ou l'avenir, elle «est» beaucoup moins; d'une part, elle n'est plus, de l'autre, elle n'est pas encore: «(...), Il s'est bien porté, Il se portera bien, ne sont pas véritablement des verbes, mais ce sont des cas du verbe; ils diffèrent du verbe en ce que le verbe indique le temps présent, tandis que les autres indiquent des temps accessoires» (ibid.: 153). Les verbes autres que le verbe substantif pris en eux-mêmes et avec leur signification propre, sans l'addition du temps et du mode, ne sont que des noms, et comme les noms «signifient un objet spécial». Ainsi comme nom et en soi un verbe, courir sans l'addition des temps et des modes, n'exprime que l'idée de course. Par lui-même, il n'a donc pas un sens complet. Il en est de même pour le verbe être: il faut un attribut, qu'il joigne au sujet, pour que la pensée soit complète: «Par lui seul le verbe n'est rien, il indique seulement, outre son sens propre, une certaine combinaison qu'on ne peut nullement comprendre indépendamment des choses qui la forment» (ibid.: 154-155).

Ce qui est donc déterminant, chez Aristote, pour qu'une unité significative soit un verbe ou un nom, c'est le fait d'être ou de ne pas être accompagné d'une modalité temporelle, ceci indépendamment de sa valeur sémantique. De plus, le verbe apparait toujours avec une expansion obligatoire, comme par exemple le sujet.

Il convient de remarquer qu'Aristote ne fut pas le seul Ancien à se pencher sur la notion de nom et celle de verbe. Les traces les plus anciennes de l'opposition verbo-nominale se trouvent dans le Sophiste (1925: 262) de Platon: «Ce qui exprime les actions (práxeis) nous l'appellerons verbe. (...) Quant aux sujets qui font ces actions, le signe vocal qui s'y applique est un nom».

Denys le Thrace, à son tour, dans l'ouvrage Tekhnē Grammatikë, la première grammaire européenne, rédigée vers les années 100 av. J.-C., semble mélanger les critères de compatibilités syntaxiques avec des critères de contenu sémantique: «Le verbe est un mot sans cas (indéclinable) susceptible d'être déterminé par des modalités aussi bien de temps que de personne et de nombre, représentant une action (quelque chose qu'on fait) ou une passion (quelque chose qu'on subit)» (cité par Clairis, 1984: 25). On voit bien que cette définition comporte, d'une part, des critères identificatoires d'une classe, tels que la possibilité de recevoir un certain type de détermination et, d'autre part, des propriétés sémantiques reconnues préférentielles. Toutefois, comme ces dernières ne sont pas exclusives pour les unités de la classe en question, elles ne constituent nullement des traits permettant de la distinguer des autres. 
L'idée de l'interdépendance des catégories de la langue et des catégories de la pensée a caractérisé non seulement les recherches linguistiques des Anciens, mais elle a persisté jusqu'aux Temps modernes. «[Au XVIII ${ }^{\mathrm{e}}$ siècle], les catégories grammaticales coïncident avec celles de la Raison, d'une raison essentialiste et universelle telle qu'on la concevait alors (...) d'où une grammaire générale selon laquelle les langues ont un fondement commun; si elles diffèrent, c'est dans l'aptitude des peuples à raisonner avec rigueur et clarté; et on peut les classer d'après la conformité de leurs structures avec celles de la logique (...)» (Guiraud, 1958: 9).

La grammaire générale classique a hérité l'analyse des parties du discours de cette longue tradition qui remonte à l'Herméneia d'Aristote. La théorie du verbe substantif jointe à l'axiome de la décomposition de la proposition en deux parties ce dont on parle et ce qui est dit de ce dont on parle - est la clé de cette grammaire.

D'après Arnaud et Lancelot de Port-Royal (1662), la proposition exprime un jugement qui comprend trois termes: le sujet, l'attribut et la liaison entre les deux est. Cette structure entraîne la répartition des mots en deux groupes: ceux qui signifient les objets de nos pensées, et les autres, qui signifient la manière de nos pensées. La première catégorie comporte outre le nom, l'article, le pronom, le participe, la préposition et l'adverbe, tandis que le verbe, ainsi que la conjonction et l'interjection, appartiennent à la seconde catégorie. Le principal usage du verbe est de signifier l'affirmation. Il est le produit de la composition du verbe substantif être avec certaines expressions des objets de nos pensées: un attribut (verbes adjectifs: par exemple Pierre marche $\rightarrow$ Pierre est marchant), un sujet, un rapport au temps. La réalité, tant morphologique que sémantique, des verbes s'échappe vers la signification des objets de nos pensées, c'est-à-dire les noms (Auroux, 1984: 14).

Un changement profond se laisse observer dans la classification des espèces de mots donnée par l'encyclopédiste Beauzée (1767). Il répartit les mots déclinables en déterminatifs et indéterminatifs. Ainsi les noms sont des mots qui expriment, déterminent les êtres, en les désignant par l'idée de leur nature, tandis que les verbes sont des mots qui expriment des êtres indéterminés en les désignant par l'idée précise de l'existence intellectuelle avec relation à un attribut. Une telle définition entraine une autonomie référentielle du nom par rapport au verbe. Beauzée note aussi que la signification de l'affirmation peut être comprise dans un nom (ibid.: 15).

Dans la grammaire de Destutt de Tracy (1803), le nom occupe la première place des classes de mots parce qu'il représente une idée existant par elle-même. «Le verbe est engendré après le nom, il est un attribut complet, il exprime l'idée qu'il représente comme existant réellement et effectivement dans un autre. Il n'y a qu'un seul verbe véritable - c'est être - parce qu'il renferme l'idée d'existence, et tous les autres ne sont verbes que pour autant qu'ils renferment ce verbe (ibid: 16)». 
D'après Guiraud, le vrai éclatement de la grammaire logique est marqué par $L a$ Pensée et la langue (1922) de Ferdinand Brunot. Ce dernier «ne part que de la pensée, ordonnant la description des faits à partir des idées (...) sans distinction entre lexique et grammaire (...)» (Guiraud, 1958: 52). Brunot rejette l'idée des catégories grammaticales.

Bien que la tendance à définir les catégories de la langue, en se servant, comme critère, de leurs valeurs sémantiques, ait persisté jusqu'au $X^{\mathfrak{c}}$ siècle, c'est déjà au Moyen Âge que certains grammairiens tentaient de réaliser une grammaire indépendante de la signification. Il s'agit surtout des tenants du courant dit grammaire spéculative qui est apparu vers la moitié du XIII' siècle à la Faculté des Arts de l'Université de Paris. Comme leur théorie a eu pour principes les modes de signifier, dont nous parlons dans la suite, ils ont été nommés Modistes à la Renaissance. On compte parmi eux Martin de Dacie, Boèce de Dacie, Jean de Dacie, Simon de Dacie (auteurs danois), Thomas d'Erfurt, Siger de Courtrai (modiste belge) (Rosier, 1983).

L'origine des modes de signifier est dans les choses. Celles-ci ont des propriétés qui peuvent exister sous trois formes, selon la sphère dans laquelle elles se trouvent. Dans la sphère des êtres, les propriétés sont les modes d'être de la chose. "La chose hors de l'intellect a de nombreuses propriétés. Elle peut en effet exister sur le mode de la disposition stable et du repos, sur le mode de l'action et de la passion, sur le mode du singulier et du pluriel, etc. Toutes ces propriétés de la chose, lorsqu'elles existent hors de l'intellect sont dites modes d'être» (Martin de Dacie, cité par Rosier, 1983: 46).

Ces mêmes propriétés peuvent exister dans la sphère de l'intellection, ce sont alors les modes d'intelliger, et dans la sphère de la signification où elles sont signifiées par la voix. Ces dernières sont les modes de signifier. L'intellect les matérialise en parties du discours et catégories grammaticales.

De même que les propriétés des choses ont trois formes d'existence selon la sphère où elles se trouvent, de même la chose elle-même en a trois: elle peut être chose existante, chose intelligée et chose signifiée. L'intellect matérialise cette chose signifiée en signe vocal - un mot (dictio). Le mot, unité de signification, renvoie donc à la chose. D'après les Modistes, je souffre, souffrant, souffrance signifient une même chose; mais ce sont trois parties du discours diffèrentes parce que ces mots ont chacun un mode de signifier distinct, renvoyant à une propriété différente de la chose. Ici il faut ajouter que les propriétés qui, liées à la chose, donneront telle ou telle partie du discours, ne sont pas nécessairement les propriétés de la chose même. La propriété de substance par exemple, d'où est tiré le mode de signifier qui constituera le nom «substantif», peut être associée à toute chose, même à celle qui réellement ne peut la comporter parce qu'elle n'existe pas hors de l'intellect, par exemple, la chose du mot rien. Celui-ci peut être conçu avec la propriété de substance, c'est-à-dire conçu sur le mode de la substance, et qui suit, signifié sur le mode de la substance. Il faut uniquement, d'une part, que ce mode de 
signifier corresponde à un mode d'être hors de l'intellect, c'est-à-dire qu'il existe une propriété de substance, et d'autre part, que la chose soit intelligible.

Comme toutes les propriétés qui sont à l'origine des modes de signifier peuvent être associées à n'importe quelle chose, il est possible de négliger la spécifité de la chose pour n'étudier que ces propriétés. C'est pourquoi la grammaire peut considérer uniquement des catégories générales, en négligeant les unités concrètes.

Munie de la potentialité d'être signifiable, la propriété de la chose deviendra une partie du discours, munie d'un mode de signifier, propriété de signifier la propriété de la chose. Ceci n'est possible que parce qu'il existe une matière en laquelle l'opération peut se réaliser, la voix, qui est la cause matérielle de l'opération de signification. L'agent de cette opération est l'intellect.

La potentialité d'être signifiable permet à la propriété de la chose d'être signifiée et à la voix de signifier la propriété de la chose, d'où résulte la distinction entre modes de signifier actifs et passifs. Ces derniers sont les propriétés des choses existant sous forme signifiée. Ils ne relèvent pas réellement de la grammaire, sauf dans leur aspect formel, qui est identique à celui des modes de signifier actifs. Ceux-ci permettent à une voix de devenir partie du discours et également fondent les propriétés qui permettent aux parties du discours d'être mises en relation avec d'autres. C'est en cela qu'ils sont les principes de la grammaire, principe de la construction.

Ainsi par exemple des modes de signifier comme celui de la disposition stable et le mode du devenir se matérialisent, par la voix, en des parties du discours soit respectivement le nom et le verbe, les deux modes étant modes de signifier essentiels, c'est-à-dire, qui définissent les parties du discours. D'autres modes de signifier, dit accidentels, correspondant à des propriétés telles que, par exemple, celle de singularité et de pluralité, se matérialisent par la voix et deviennent les accidents des parties du discours, nombre, genre, personne, cas, etc. Ainsi, les catégories grammaticales essentielles et accidentelles voient leur existence confirmée et leur définition justifiée par un même principe: le mode de signifier.

Les Modistes font la distinction entre les opérations: celle qui, à partir d'une propriété de la chose signifiable et de la voix, forme une partie du discours ou un accident grammatical (opération de consignification) et l'autre qui, à partir de la chose signifiable et de la voix, forme une unité linguistique significative, un signe vocal, un mot (dictio) (opération de signification). Le mot est l'unité significative, celle du logicien, et la partie du discours est l'unité fonctionnelle, celle du grammairien. Le mot ne peut être l'unité de base de la grammaire. Il renvoie à la chose et n'a pas de correspondant linguistique unique: «Souffrance, je souffre, souffrant, douloureusement, aïe, etc. signifient la même chose» (Jean de Dacie cité par Rosier, 1983: 57).

Ce n'est qu'en tant que la chose est associée à telle ou telle propriété, donc qu'elle est signifiée sur tel ou tel mode, que l'entité linguistique, munie d'un mode de signifier actif, aura une existence pour la grammaire. Comme la chose, qui est à 
l'origine de la signification du mot, est indépendante de la propriété qui lui est associée dans l'intellect et qui est à l'origine du mode de signifier du mot, et que toute chose peut être signifiée sur n'importe quel mode, on peut négliger la spécifité de la chose, c'est-à-dire la signification. Les parties du discours sont donc définies par leur modes de signifier et non par leur signification.

Il est intéressant de comparer la typologie des modes de signifier avec celle des catégories grammaticales décrites par les ouvrages contemporains. Prenons comme exemple les modes de signifier accidentels du nom et du verbe (Rosier, 1983: 105 et 118):

\section{NOM}

I. Modes de signifier accidentels absolus.

1. Mode de signifier la chose dans son essence première ou seconde espèce:

- primitive, - dérivée.

2. Propriété de simplicité, de composition, de sur-composition - FIGURE:

- simple,

- composée,

- surcomposée.

II. Modes de signifier accidentels relatifs.

1. Mode de signifier la chose sous la propriété de l'action ou de la passionGENRE:

- masculin,

- féminin,

- neutre.

2. Propriété d'indivisibilité ou de divisibilité - NOMBRE:

- singulier,

- pluriel.

3. Propriété de principe ou de terme - CAS (6 cas):

4. Propriété de parler - PERSONNE:

- première,

- seconde,

- troisième.

\section{VERBE}

I. Modes de signifier accidentels absolus.

1. Mode de signifier la chose dans son essence première ou seconde - FORME:

- primitive (je lis),

- dérivée (p.ex.: fréquentative, inchoative, diminutive).

2. TEMPS:

- présent, 
- passé,

- futur.

II. Modes de signifier accidentels relatifs.

1. Propriété d'inhérence selon l'être, qui permet au verbe de se rapprocher du sujet - COMPOSITION.

2. MODE (qualité de la COMPOSITION):

- mode de l'indication,

- de la commande,

- du souhait,

- du doute,

- de l'indéfinition (infinitif).

3. SIGNIFICATION.

4. VOIX (qualité de la SIGNIFICATION).

5. Propriété de parler du sujet - PERSONNE.

Avec le développement des recherches linguistiques à l'époque moderne, la thèse que les mécanismes qui régissent l'utilisation de la langue prendraient leur source dans les lois universelles communes à tous les hommes, trouvait de plus en plus d'adversaires. L'étude de la structure de nombreux langages très différenciés a montré que la caractéristique dominante de l'idiome n'était pas qu'il manifestait l'universalité de l'esprit humain. Tout au contraire, d'énormes et multiples différences qui séparent les systèmes ont été mises en évidence. Ce qui était frappant, c'est le caractère arbitraire et conventionnel des catégories dégagées par l'analyse et la complexité des règles syntactiques limitant les possibilités combinatoires des éléments. Ainsi donc, la logique semblait avoir peu de rapport avec ce qu'était une langue. Déjà au XVIII ${ }^{\mathrm{e}}$ siècle, la linguistique moderne établissait le caractère essentiellement alogique du langage (Guiraud, 1958: 9).

La linguistique structurale rompt d'une manière radicale avec les méthodes traditionnelles de recherches en renonçant définitivement à la subordination des catégories grammaticales à celles de la pensée. Ces premières doivent être établies, d'après les structuralistes, uniquement à partir des critères formels, c'est-à-dire, pratiquement, à partir d'une analyse des oppositions décelables dans le signifiant linguistique. Ainsi, les classes de formes sont déterminées par le seul moyen des relations de compatibilité ou d'incompatibilité contractées par les éléments (Corneille, 1976: 67).

Dans ses Principes de grammaire générale Louis Hjemslev insiste sur le fait que «l'idée purement »intérieure«, l'idée psychologique proprement dite, (...) ne concerne pas la grammaire». D'après lui, c'est l'association du signifiant et du signifié qui constitue le «critère indispensable et infaillible par lequel on peut reconnaître ce qui est de la grammaire et ce qui n'en n'est pas». Une catégorie qui n'est pas fondée sur des critériums de forme ne saurait lui appartenir (Hjemslev, 1928: 27). L'auteur présente, dans le même ouvrage, la justification de son point de vue. Relisons, à titre d'exemple, cette citation: «Etablir des catégories purement 
psychologiques sans critériums de forme est chose facile et qui n'exige guère de réflexion, mais qui est aussi dénuée de tout intérêt au point de vue grammatical. On pourrait par exemple très aisément établir une catégorie de mots désignant des objets noirs (ou noirâtres), tels que charbon, graphite, ébène, nègre, nuit, encre, etc. Il n'y a pas de différence essentielle entre une telle catégorie (...) et, par exemple, telle autre catégorie composée de "mots signifiant une action «. (...) Les catégories purement sémantiques ou psychologiques que l'on vient à établir n'ont jamais de chance de coïncider avec des catégories vraiment grammaticales» (ibid.: 29-30). La conclusion de l'auteur est la suivante: «Les catégories linguistiques ne peuvent pas être des calques purs et simples des catégories logiques». Elles sont propres au langage et «ne se retrouvent nulle part en dehors de son domaine» (ibid.: 78 et 80).

Il est facile d'apercevoir une concordance d'avis chez d'autres linguistes par rapport à la question susmentionnée. Citons par exemple Edward Sapir: «Une "partie du discours « mise en dehors des restrictions de la syntaxe n'est qu'une vapeur insaisissable; pour cette raison, aucun tableau logique des "parties du discours « (...) ne peut être d'un intérêt quelconque pour le linguiste; chaque langage a son tableau personnel, et tout dépend des démarcations qu'on reconnaît entre les formes» (Language, 1921: 115; trad. J-P.Corneille). L. Bloomfield, à son tour, complète sa critique des Anciens évoquée plus haut, par la constatation suivante: «Ils les ont définies [parties du discours, constructions syntaxiques et principales catégories inflexionnelles] non pas en termes de formes linguistiques reconnaissables mais en termes abstraits qui devaient indiquer la signification de la catégorie linguistique» (Bloomfield, 1970: 11). Il résulte des citations ci-dessus que les structuralistes établissent les catégories de la langue (les classes de formes) indépendamment de toute référence à la logique ou à la psychologie. Ils ne prennent en considération que les contraintes spécifiques du code étudié. Ici ajoutons encore la constatation de deux descriptivistes américains, Bernard Bloch et George Trager, comprise dans leur ouvrage Outline of linguistic analysis: «Toutes nos classifications doivent se fonder exclusivement sur la forme - sur des différences et des similitudes dans la structure phonématique des racines et des affixes - ou sur des occurrences de certains mots dans certains types particuliers de propositions et d'énoncés (...). Les définitions traditionnelles des parties du discours, telles que nous les avons apprises à l'école, se fondent largement sur des considérations philosophico-sémantiques. »Le nom est un mot utilisé pour désigner un être ou une chose«. Une telle définition est dépourvue d'intérêt; elle ne nous apprend rien concernant la structure de la langue anglaise et ne nous permet pas de reconnaître un nom lorsque nous en rencontrons un» (cité par Corneille, 1976: 69).

Non seulement les parties du discours, mais de même toutes les autres catégories grammaticales - comme le genre, le nombre, le temps, l'aspect, le mode, etc. - doivent être considérées comme des catégories purement formelles établies en fonction des contraintes spécifiques du code envisagé, indépendamment de leur contenu de sens. Il peut arriver que des classements formels recouvrent, du moins partiellement, des classements notionnels, mais l'essentiel est de ne jamais 
subordonner les premiers aux seconds. Les critères de forme doivent avoir une absolue priorité sur les critères sémantiques.

Afin de l'illustrer, servons-nous de l'exemple tiré d'un ouvrage de Jean Dubois, et concernant la répartition des substantifs en deux catégories: animés et inanimés. Dans le premier groupe, l'auteur met en relief la distinction entre la catégorie de noms d'animaux qui connaît la variation de marque de genre et une autre qui ignore cette distinction: le lion/la lionne, le tigre/la tigresse, et de l'autre côté, le moustique, la girafe, la fourmi. Il est facile de remarquer que l'emploi du critère sémantique, dans ce cas, mène à classer ces mots dans la même catégorie (Dubois, 1965: 54).

Dubois insiste sur la nécessité de distinguer le code en tant que tel de l'utilisation qui en est faite. La confusion des plans - sémantique et formel - a pour inconvénient de masquer le fonctionnement réel du système et de cacher les relations fonctionnelles entre ses éléments: «Dans un code défini, un système de marques reçoit une exploitation sémantique dominante, avec laquelle on a tendance à le confondre; ainsi, on identifie l'opposition de marque de nombre avec l'opposition singularité/pluralité, ou l'opposition de genre avec celle de sexe; mais c'est confondre deux plans différents, c'est méconnaître que, s'il y a correspondance entre un ensemble de signifiés et un ensemble de signifiants, il n'y a pas identité. (...) dans la catégorie I [inanimés], la fonction du genre est purement classificatoire. (...) l'exploitation dominante de l'opposition dans la catégorie A [animés] ne saurait intéresser la catégorie $I$ et (...) ainsi le système de marques devient disponible pour d'autres fonctions" (ibid.: 89).

L'étude des catégories de temps et d'aspect conduit l'auteur à la conclusion qu'il est nécessaire d'envisager à l'aide de quelles structures formelles sont traduites les oppositions sémantiques que l'on appelle, d'une part, l'aspect, et de l'autre, le temps. Une telle étude comporte l'établissement des moyens mis en œuvre, indépendamment de l'exploitation dont elles sont l'objet et par la seule détermination des rapports entretenus par les formes. «Le problème linguistique est de savoir si, les classes de formes une fois définies par la spécificité de leurs marques et par les distributions dans les phrases des segments qui les composent, il en existe une ou plusieurs qui soient utilisées, de préférence aux autres, pour servir de base formelle à ces oppositions de signifiés» (Dubois, 1964: 3).

D'après John Lyons, le structuralisme est le trait le plus marqué de la linguistique moderne. Le terme lui-même signifie que toute langue est considérée comme un système de relations ou comme un ensemble de systèmes reliés les uns aux autres, dont les éléments n'ont aucune valeur indépendamment des relations d'équivalence et d'opposition qui les relient (Lyons, 1968: 41). Une telle conception implique qu'il n'existe aucun lien nécessaire immédiat entre la structure interne du langage (relationnelle et conventionnelle), d'une part, et la logique, la pensée ou la réalité extra-linguistique, d'autre part. Les catégories objectives dégagées par l'analyse seront donc établies exclusivement en fonction des relations contractées par les éléments de la structure, sur la base de critères purement 
formels, indépendamment de tout schème logique, psychologique ou sémantique préétabli.

La description des catégories de la langue proposée par les structuralistes paraît toutefois avoir des insuffisances tant sur le plan morphologique que syntaxique. Il résulte des deux exemples cités ci-dessous que la linguistique ne peut se désintéresser totalement des problèmes soulevés par la logique.

Le premier exemple, présenté par André Martinet dans La Linguistique. Guide alphabétique comprend deux phrases apparemment identiques au point de vue de la syntaxe mais qui ne peuvent recevoir un traitement identique sur le plan de la logique:

Ce drapeau est blanc et large,

et

Ce drapeau est blanc et bleu.

L'auteur complète les deux phrases par le commentaire suivant: «De l'énoncé Ce drapeau est blanc et large, on peut inférer qu'il est blanc. Mais on ne peut pas tirer la même conclusion à partir de Ce drapeau est blanc et bleu. Il peut être intéressant pour le linguiste de chercher à expliquer ce fait. S'agit-il d'une homonymie et y a-t-il en français deux $e t$ ? Ou bien les deux énoncés ont-ils des constructions grammaticales différentes, susceptibles d'être décrites au niveau de l'analyse syntaxique? Ou encore doit-on faire intervenir le fait que blanc et bleu sont tous deux des adjectifs de couleur, et admettre que la langue française possède une catégorie sémantique «adjectif de couleur»? Même si le linguiste devait finalement reconnaître que la réponse à ce problème n'est pas de sa compétence, il reste (...) qu'en décidant de n'y pas répondre, le linguiste est amené à définir plus nettement les limites de sa compétence» (Martinet, 1969: 239).

Comme deuxième exemple, confrontons les syntagmes suivants:

des beignets aux pommes froids

et

des tartes aux amandes fraîches.

(cité par Corneille, 1976: 73)

Les deux présentent la même suite des constituants (dét. $+\mathrm{N}_{1}+$ prép. $+\mathrm{N}_{2}+$ Adj.), et pourtant, malgré cette apparence extérieure identique, le deuxième syntagme, par opposition au premier, peut recevoir deux interprétations distinctes, l'adjectif pouvant se référer, en fonction du contexte, tant au premier qu'au deuxième nom.

Les deux exemples confrontés avec les méthodes des structuralistes font preuve que ces derniers décrivent une langue objet, et non la manière dont celle-ci peut être utilisée dans le raisonnement. Leurs techniques qui consistent à analyser 
des propriétés formelles réalisées dans les énoncés ne leur permettent pas de résoudre des questions comme celles qui découlent des deux exemples ci-dessus.

Le désaccord entre la grammaire et la logique consiste, d'après J. Vendryès (1968:132), en ce que les catégories grammaticales et les catégories logiques se recouvrent très rarement. Quand on essaie de mettre de l'ordre dans les faits grammaticaux en les classant d'après la logique, on est conduit à faire une répartition arbitraire: tantôt on rangera en des catégories logiques distinctes des faits qui ont le même aspect grammatical (ce qui est, pour l'auteur, faire violence à la langue), tantôt on laissera groupés sous la même catégorie grammaticale des faits qui n'ont logiquement rien de commun (ce qui est, d'après lui, faire violence à la raison). Il est pourtant hors de doute que, bien qu'elles puissent varier suivant les langues, les catégories grammaticales ont en effet, dans la langue où elles règnent, un pouvoir qui domine l'activité de l'esprit. De plus, quelles que soient les différences des habitudes mentales chez les différents peuples, l'existence de certains traits fondamentaux n'est pas niable. Il y a une logique humaine et de grandes catégories logiques se retrouvent chez tous les hommes qui pensent. Elles sont, d'après l'auteur, à la base des catégories grammaticales.

\section{BIBLIOGRAPHIE}

Aristote, (1932), Poétique, trad. J. Hardy, Paris.

Aristote, (1943), Herméneia, in: Logique d'Aristote, v. I., trad. J. Barthélemy Saint-Hilaire, Paris.

Aristote, (1989), Catégories, in: Organon, trad. J. Tricot, Paris, pp. 1-76.

Auroux S., (1984), Du nom au verbe: la grammaire générale de Port-Royal à Destutt de Tracy, Modèles Linguistiques, t. VI, fasc.1, Villeneuve d'Ascq, pp. 11-20.

Benveniste E., (1966), Problèmes de linguistique générale, Paris.

Bloomfield L., (1970), Le Langage, trad. J. Grazio, Paris.

Clairis Ch., (1984), Nom et verbe, Modèles Linguistiques, t. VI, fasc.l, Villeneuve d'Ascq, pp. 23-28.

Corneille J.-P., (1976), La linguistique structurale, sa portée, ses limites, Paris.

Dubo is J., (1964), La Traduction de l'aspect et du temps dans le code français (Structure du verbe), Le Français Moderne 1, Paris, pp. 1-26.

Dubois J., (1965), Grammaire structurale du français. Nom et pronom, Paris.

Dubois J. et coll., (1973), Dictionnaire de linguistique, Paris.

Guiraud P., (1958), La Grammaire, Paris.

Hjems lev L., (1928), Principes de grammaire générale, Copenhague.

Lyons J., (1968), Linguistique générale. Introduction à la linguistique théorique, trad. F. Dubois-

-Charlier et D. Robinson, Paris.

Martinet A., (dir.), (1969), La Linguistique, Guide alphabétique, Paris.

Platon, (1925), Sophiste, trad. A. Diès, Paris.

Rey A., (1973), Théorie du signe et du sens, Paris.

Rosier I., (1983), La Grammaire spéculative des Modistes, Lille.

Sapir E., (1921), Language, An Introduction to the Study of Speech, New York.

Vendryěs J., (1968), Le Langage, Introduction linguistique à l'histoire, Paris. 